\title{
Effects of Jute Fibres Percentage Variation on Cotton-Jute Blended Fabric: Evaluation of Blended Fabrics After Dyeing Process
}

\author{
Md. Solaiman ${ }^{1, ~ *, ~ K h a i r u l ~ I s l a m ~}{ }^{1}$, Sanoar Hossain ${ }^{1}$, Moshiur Rahman ${ }^{1}$, Mahfuz Alam, \\ Ataur Rahman", Abul Kasem Mohammed Fazlul Haque ${ }^{2}$ \\ ${ }^{1}$ Department of Fabric Manufacturing Engineering, Textile Engineering College, Noakhali, University of Textiles, Dhaka, Bangladesh \\ ${ }^{2}$ Department of Yarn Manufacturing Engineering, Textile Engineering College, Noakhali, University of Textiles, Dhaka, Bangladesh
}

\author{
Email address: \\ solaimanbari@gmail.com (Md. Solaiman), tex.khairul@gmail.com (K. Islam), sanowarnipun@gmail.com (S. Hossain), \\ moshiurtex97@gamil.com (M. Rahman), mas.shojib@gmail.com (M. Alam), akmfazlulhaque1@gmail.com (A. K. M. F. Haque) \\ ${ }^{*}$ Corresponding author
}

\section{To cite this article:}

Md. Solaiman, Khairul Islam, Sanoar Hossain, Moshiur Rahman, Mahfuz Alam, Abul Kasem Mohammed Fazlul Haque. Effects of Jute Fibres Percentage Variation on Cotton-Jute Blended Fabric: Evaluation of Blended Fabrics After Dyeing Process. International Journal of Industrial and Manufacturing Systems Engineering. Special Issue: Manufacturing Strategy for Competitiveness. Vol. 4, No. 6, 2019 , pp. 64-70. doi: $10.11648 /$ j.ijimse.20190406.12

Received: September 16, 2019; Accepted: October 15, 2019; Published: December 18, 2019

\begin{abstract}
In this study, two fabrics were collected from jute research institute, Bangladesh consisting of $80 \%$ Jute \& $20 \%$ cotton yarn; $50 \%$ Jute and $50 \%$ Cotton yarn by weight, to make comparison between the samples. The tearing strength (TS), drape coefficient, Pilling resistance, Color fastness to wash \& rubbing, water take up $\%$ and Color strength were investigated. From analyzed data, it was found that tearing strength in warp and weft way direction is higher for $80 \%$ jute and $20 \%$ cotton blended fabric. From results it was seen that in warp direction, tearing strength for $80 \%$ and $20 \%$ cotton blended fabric is $4 \%$ higher than $50 \%$ jute and $50 \%$ cotton blended woven fabric but in weft direction $356 \%$ higher for $80 \%$ jute and $20 \%$ cotton blended woven fabric. Color fastness to rubbing is comparatively better for $50 \%$ jute and $50 \%$ cotton blended sample. Wash fastness property is almost same for both blended samples. But jute prominence sample carried poor wash fastness property on blended cotton and polyamide in compare equally blended sample. Moderate pills are formed on 50\% cotton and 50\% jute blended fabric and slight surface fuzzing and/ or partially formed pills on $80 \%$ jute and $20 \%$ cotton blended sample. Drape resistance property is better for jute prominence blended fabric. Color strength is almost same for both samples.
\end{abstract}

Keywords: Cotton, Jute, Woven Fabric, K/s Value, Reactive Dye

\section{Introduction}

Fabric or cloth is the second basic needs of human being after food. Since the beginning of civilization weaving of cloth has been carried out in one form or the other by people of many countries. A woven cloth consists of two sets of yarns, namely warp and weft. The yarns that are placed lengthwise or parallel are called warp yarns and the yarns that run crosswise are called weft yarns [1].

In recent times, various types of woven fabrics are observed throughout the market place. It varies from styles, designs, compositions, structures, etc. To develop the criteria and quality of woven fabrics, blends of two or more types of fibre are used. A fabric which is produced from the blended yarn might have better characteristics than what could be obtained in a fabric produced from a single fiber.

Jute is one of the world's most important fibrous crops, being exceeded in quantity only by cotton. It has long been known to people as a golden fiber. Jute fiber is a bast fiber obtained from the bark of jute plant containing three main categories of chemical compounds namely cellulose (58 63\%), hemicellulose (20-24\%) and lignin (12 15\%), and some other small quantities of constituents like fats, pectin, aqueous extract, etc. Jute fiber is composed of small units of cellulose surrounded and cemented together by lignin and hemi-cellulose [2]. 
Cotton is a soft, fluffy white staple fiber which grows in a boll or protective case, around the seeds of the cotton plants of the genus Gossypium in the mallow family Malvaceae.

In the present work, a few characteristics of jute-cotton blended fabrics have been evaluated. Fabric produced from the blended yarn might have better characteristics than what could be obtained in a fabric produced from a single fiber. The blending of cotton is done to develop drape properties, comfortability, durability, dyeability and many other properties of the fabric products. Any successful attempt to blend jute fiber with cotton would be a breakthrough in the field of textile [3].

Blending jute with cotton fiber may be an acceptable way of jute diversification by which value added products can be produced. Jute fibers have several advantages like a lustrous golden appearance, high tenacity and good properties. Hence the techniques of blending and softening could as utilized to upgrade the quality of jute and thus form a new class of jute-based fabrics having an expanding market within and outside the country [4].

As jute is an available and cheap fibre, it may be provide a great support to our national economy to reduce raw materials import cost of fabric. Jute-Cotton blended fabric may also lead to environmental awareness because of having environmental friendly characteristics of Jute. To develop better quality of fabric and reduce production cost by using jute cotton blended yarn as a raw material is a very much beneficial suggestion for upcoming decades.

\section{Materials}

For this experiment two samples of Cotton -Jute blended woven plain fabrics were used where one sample have cotton and jute percentage were $20 \%$ and $80 \%$ respectively and another have $50 \%$ jute $\& 50 \%$ cotton respectively. The final target of this work is to analysis the effect of jute fibres percentage variation on cotton-jute blended fabric. Samples were collected from Bangladesh jute Research Institute. The samples (2/2 Plain structure and 1/1 Plain structure) weighs were $465 \mathrm{gm} / \mathrm{m}^{2}$ and $230 \mathrm{gm} / \mathrm{m}^{2}$ respectively with warp density 86 ends/inch \& 49 ends/inch respectively and weft density 25 picks/inch \& 39 picks/inch respectively. Fastness Tester, Beesley's balance, Light fastness Tester, Crocking meter, Stop watch, crease recovery tester, Tensile and Tearing strength tester and Spectrometer were used to do the physical, mechanical and visual properties of the fabrics.

\section{Methods of the Experiments}

\subsection{Scouring and Bleaching Procedure of Blended Fabric}

Set the bath with substrate at room temperature with wetting agent, soda ash $\& \mathrm{NaOH}$. Then added sequestering agent and raised the temperature to $100^{\circ} \mathrm{C}$. After reaching $100^{\circ} \mathrm{C}$ temperature added stabilizer, $\mathrm{H}_{2} \mathrm{O}_{2}$ and run 45 minutes at the same temperature. After that cooled the bath and then drain out water with ingredients. Then rinsed the samples two times with hot and cold water respectively. Finally treated the samples with a peroxide killing agent for 10 minutes to remove the residual peroxide and then rinsed the samples with cold water again.

\subsection{Dyeing Procedure of Blended Fabric}

Jute fibre has a special affinity to basic dyestuff but cotton fibre has a great affinity to reactive dyes for better fastness properties. For this reason, the specimens are dyed with basic dye and reactive dye. To increase the affinity of cotton towards basic dye, mordanting was done.

\subsection{Mordanting Process}

Dye bath was set at room temperature, then raised the temperature at $100^{\circ} \mathrm{C}$. Then tannic acid was added and reduced the temperature, and fixed temperature at $60^{\circ} \mathrm{C}$ and run the machine for 2 hours and then drained out the liquid. After mordanting, fixation was done. For fixation tartaric acid was added at room temperature and run the machine for 30 minutes and dropped the liquor and rinsed the samples.

\subsection{Basic Dye Application Procedure to Specimen}

Firstly, made paste by adding water, wetting agent and acetic acid with dyes; then added hot water and stirred to make desirable dye solution. Set the dye bath with substrates at room temperature. Added 1/3 of total amount of paste to the bath at $25^{\circ} \mathrm{C}$ and run the bath 15 minutes. Then raised the temperature $40^{\circ} \mathrm{C}$, again added $1 / 3$ of total paste and run the machine for 20 minutes. After that $1 / 3$ of remaining paste is added at $70^{\circ} \mathrm{C}$ and run the machine for 25 minutes. Then cooled the bath and drained out liquor.

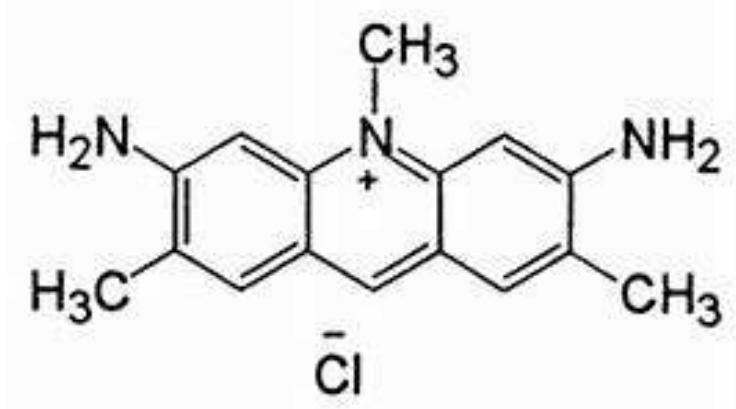

Figure 1. Chemical structure of basic dye.

\subsection{Reactive Dye Application Procedure to Specimen}

Set the dye bath with substrate at room temperature. Added wetting agent, Sequestering agent, leveling agent and dye at room temperature and raised the temperature to $80^{\circ} \mathrm{C}$. Run the bath for 10minutes then added half of total Glauber salt and after 10 minutes residue half portion of salt was added. After that Soda ash was added at the same temperature in two stages and then run the machine for 25 minutes. Dropped the liquor and rinsed two times by cold and hot wash with soap and soda. 


\section{Testing and Analysis}

\subsection{Testing Procedure Tear Strength (ISO 13937-2 \& ASTM D1424)}

Cut the samples $(80 \mathrm{~cm} \times 58 \mathrm{~cm})$ according to the template from left, middle and right. Place the pendulum at the notch point and put the samples in the jaw and clamp it $(80 \mathrm{~mm}$ is parallel to horizontal \& $58 \mathrm{~mm}$ is vertical direction). Notch the sample by sharp knife $(15 \mathrm{~mm})$. Release the pendulum by depressing pendulum stop point to sewing a full cycle for tearing the fabric before releasing the stop button. [5].

\subsection{Testing Procedure Colorfastness to Rubbing (ISO 105X12; 1993; BS EN ISO 105X12; 1995)}

\section{Test Specimen:}

Two pieces $14 \mathrm{~cm} \times 5 \mathrm{~cm}$ specimens are required for dry rubbing and two for wet rubbing.

Test Procedure:

Dry Rubbing Test:

Lock the test specimen onto the base of the Crock meter so that it lies flat and taut for test and taut for testing. Using the spiral clip, set a $5 \mathrm{~cm} \times 5 \mathrm{~cm}$ square of the white cotton, rubbing test cloth to set the finger of the Crockmeter. Lower the covered finger. Turn the hand crank and make ten complete turns of the crank, at the rate of one turn per second. Remove rubbing test specimen cloth from the finger and evaluate colour transfer using the gray scale for staining under standard lighting, D65. One test is done to warp /wales direction and another for weft / course direction. [6, 7]

Wet Rubbing Test:

Repeat the procedure on another sample with the white test cloth wetted in distilled water. Ensure that the rubbing test cloth will be wetted with water to $100 \%$ take up. Air dries the cotton-jute blended test cloth at the room temperature before evaluation.

\subsection{Testing Procedure of Color Fastness to Washing (ISO 105 C06)}

Collect the samples $(10 \mathrm{~cm} \times 4 \mathrm{~cm})$ from bulk and then conditioning for 04:30 hours to 06 hours. Make a specimen of $4 \mathrm{~cm} \times 10 \mathrm{~cm}$ in size. Sew the specimen with multi-fiber fabric $(10 \mathrm{~cm} \times 4 \mathrm{~cm})$ of same size at one corner. Make the solution of $4 \mathrm{gm} /$ litre ECE detergent \& $1 \mathrm{gm} /$ litre sodium perborate. Put the specimen with multi-fibre fabric into the solution in Rotary washing $\mathrm{m} / \mathrm{c}$ and taking temp: $60^{\circ} \mathrm{C}$ at $30 \mathrm{~min}$ with 25 pcs still ball. Rinse with hot water respectively. Squeeze with cold water of the sample is done (Hand Wash). Then drying is done at a temperature in the air not exceeding $60^{\circ} \mathrm{C}$. The stitching is then broken out except on one of the shorter end. Measure the staining and color change by grey scale $\&$ make a test report. [8]

\subsection{Testing Procedure of Pilling Test (ICI Pilling Box) (ISO 12945-1)}

The sample (5" x 5") is sewn so as to be firm fit when placed a rubber tube 6" long, 1.25 " outer diameter and 0.125 " thick. The cut ends of the fabric are covered by cellophane tape and 4 tubes are placed in a box (9"x 9" $\mathrm{x} 9$ 9") lined with cork 0.125 " thick. The box containing the tube is then rotated at $60 \mathrm{rpm}$ for 5 hours. After tumbling, the extent of pilling assessed visually comparison with the arbitrary standards. [9]

\subsection{Testing Procedure of Drape Co-efficient (ISO 9073-9: 2008)}

\section{Test Specimen:}

Make samples at a dimension of $30 \mathrm{~cm}$ diameter.

Test Procedure:

In the check a circular specimen is command concentrically between 2 smaller horizontal discs and is allowed to drape into folds beneath its own weight. A light-weight is shone from beneath the specimen and also the shadow that the material casts, is copied onto aringed piece of paper identical size because the unsupported a part of the material specimen. To measure the areas concerned, the total paper ring is weighed then the shadow part of the ring is cut away and weighed. The paper is assumed to possess constant mass per unit spaceso the measured mass is proportional to space. [10]

\subsection{Testing Procedure of Color Strength}

The colour intensities of $50 \%$ Jute \& $50 \%$ Cotton blended woven fabric and $80 \%$ Jute \& $20 \%$ Cotton blended woven fabric for a specific design and construction, fabrics are measured and compared using a UV VIS-NIR reflective spectrometer (Spectra Flash SF 600 X, USA), over the range of $360 \mathrm{~nm}$ to $700 \mathrm{~nm}$.[11, 12]

\subsection{Testing Procedure of Water Takes Up Percentage (ASTM D-570)}

Test Specimen:

Make a sample at a dimension of $2 \mathrm{~cm}$ in length $\mathrm{x} 1 \mathrm{~cm}$ in width.

Test Procedure:

Samples were immersed in a beaker containing $100 \mathrm{ml}$ of deionized water at room temperature for 1 hour. Initially, weight of samples was determined, after certain time interval; samples were taken out of the beaker and wiped using tissue papers. Their weight was taken again. In this case, it shows no uptake after 40 minutes; that why carried out the test upto 1 hour water uptake percentage was determined by using following equation-

Water up-take $=[$ Wet weight - Dry weight $) /$ Dry weight $\mathrm{x}$ $100[12,13]$

\section{Results and Discussion}

\subsection{Determination of Tearing Strength}

The Elmendorf tear test determines the tearing strength by measuring the work done in tearing through a fixed length of the test specimen. It is usually measured in $\mathrm{kN} / \mathrm{m}$ or pounds (lbf).

As it can be seen from Figure 2, that tearing strength for warp way direction of $80 \%$ Jute \& $20 \%$ Cotton blended 
woven fabric is greater than $50 \%$ Jute \& $50 \%$ Cotton blended woven fabric after dyeing. From Figure 3, it is also seen that tearing strength for weft way direction of $80 \%$.

Jute \& $20 \%$ Cotton blended woven fabric is many times higher than 50\% Jute \& 50\% Cotton blended woven fabric. The strength of Jute is greater than Cotton. For this reason $80 \%$ Jute \& $20 \%$ Cotton blended woven fabric possess a high tearing strength.

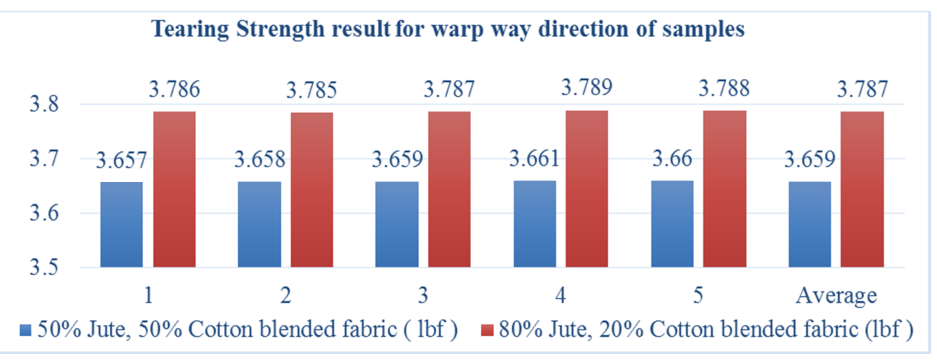

Figure 2. Tearing strength results in warp way direction of samples.

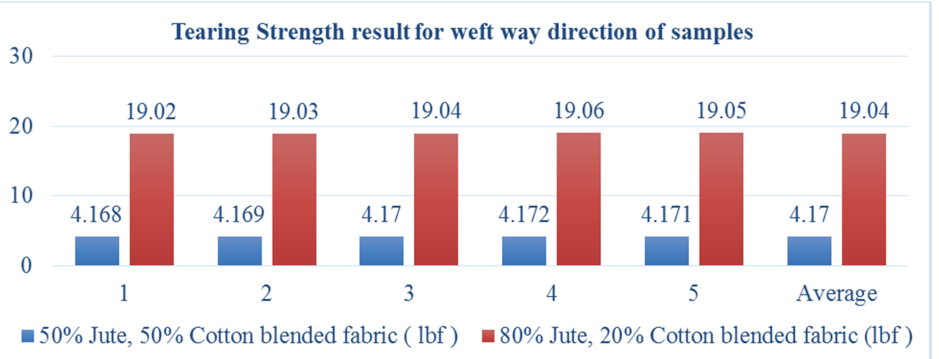

Figure 3. Tearing strength results in weft way direction of samples.

\subsection{Determination of Colorfastness to Rubbing}

From table 1, compare the contrast between untreated and treated white rubbing cloth with the staining Grey Scale and rate from 1 to 5 and changing in the tested specimen with changing Grey Scale. This test is designed to determine the degree of colour which may be transferred from the surface of a coloured fabric to specify test cloth for rubbing (which could be dry and wet). From the both samples, it is seen that amount of staining moderate at dry condition and at the wet condition, the amount of staining is higher than dry condition rubbing. As basic dyes are poor to moderate fastness properties, so the rubbing fastness of $50 \%$ Jute \& $50 \%$ Cotton blended woven fabric is better than $80 \%$ Jute \& $20 \%$ Cotton blendedwoven fabric.

Table 1. Colorfastness to rubbing results for specimens.

\begin{tabular}{lllll}
\hline \multicolumn{2}{l}{ Dry condition Rubbing result } & Wet condition Rubbing result \\
\hline \multirow{2}{*}{ SI. No } & $\begin{array}{l}\mathbf{5 0 \%} \text { Jute \& } \mathbf{5 0 \%} \text { Cotton } \\
\text { blended woven fabric }\end{array}$ & $\begin{array}{l}\mathbf{8 0 \%} \text { Jute \& } \mathbf{2 0 \%} \text { Cotton } \\
\text { blended woven fabric }\end{array}$ & $\begin{array}{l}\mathbf{5 0 \%} \text { Jute \& 50\% Cotton } \\
\text { blended woven fabric }\end{array}$ & $\begin{array}{l}\mathbf{8 0 \%} \text { Jute \& 20\% Cotton } \\
\text { blended woven fabric }\end{array}$ \\
\hline 1 & 4 & $3-4$ & 3 & $2-3$ \\
2 & 4 & 3 & $2-3$ & $2-3$ \\
3 & $4-5$ & $3-4$ & 3 & $2-3$ \\
Average & 4 & $3-4$ & $2-3$ \\
\hline
\end{tabular}

\subsection{Determination of Color Fastness to Washing}

From the tables 2 and 3, it is found that dyes stained on the fibres of the multifibre fabric show the rating are almost same for both fabrics. The staining on the di-acetate, nylon, polyester, acrylic, wool for 50\% Jute \& 50\% Cotton blended woven fabricis found in good rating value that is 4 to 5, which shows moderate staining and for bleached Cotton, staining rating is 2-3 which indicate lower staining. In case of $80 \%$ Jute \& $20 \%$ Cotton blended woven fabric, the staining rating for Di-acetate, Polyester, Acrylic and wool are moderate and for Bleached Cotton and Polyamide rating is 2-3 i.e. lower staining because of prominence of jute is higher.

Table 2. Results of Color Fastness to wash of $50 \%$ Jute \& $50 \%$ Cotton blended woven fabric.

\begin{tabular}{lllll}
\hline Multifibre's layer & Sample 1 & Sample 2 & Sample 3 & Average \\
\hline Di-acetate & 4 & 4 & 4 & $2-3$ \\
Bleached Cotton & 3 & $2-3$ & 4 & $2-3$ \\
Polyamide & $3-4$ & 4 & $4-5$ & $3-4$ \\
Polyester & $4-5$ & $4-5$ & $4-5$ & $4-5$ \\
Acrylic & $4-5$ & $4-5$ & 4 & 4 \\
Wool & 4 & 4 & 4 & 4 \\
\hline
\end{tabular}


Table 3. Results of Color Fastness to wash of $80 \%$ Jute \& $20 \%$ Cotton blended woven fabric.

\begin{tabular}{lllll}
\hline Multifibre layers & Sample 1 & Sample 2 & Sample 3 & Average \\
\hline Di-acetate & 4 & 4 & 4 & 4 \\
Bleached Cotton & $2-3$ & $2-3$ & 3 & $2-3$ \\
Polyamide & $2-3$ & 3 & $2-3$ & $2-3$ \\
Polyester & $4-5$ & $4-5$ & $4-5$ & $4-5$ \\
Acrylic & 4 & 4 & 4 & 4 \\
Wool & $3-4$ & 4 & $3-4$ & $3-4$ \\
\hline
\end{tabular}

\subsection{Determination of Pilling Resistance}

Pilling is a fabric surface characterized by little pills of entangled fiber clinging to the cloth surface and giving the garment unsightly appearance. The pills are formed during wear and washing by the entanglement of loose fibers which protrude from the fabric surface Pilling resistance property for the treated and untreated sample is remaining almost unchanged.
From figure 4 , it is seen that the rating of pilling is 3, which indicate moderate surface fuzzing or moderate pilling for $50 \%$ Jute \& 50\% Cotton blended woven fabric and for $80 \%$ Jute \& $20 \%$ Cotton blended woven fabric rating is 4 , which indicate slight surface fuzzing or partially formed pills. The higher percentage of small projecting fibre presents in 50\% Jute \& $50 \%$ Cotton blended woven fabric is reason behind this variation of pilling.

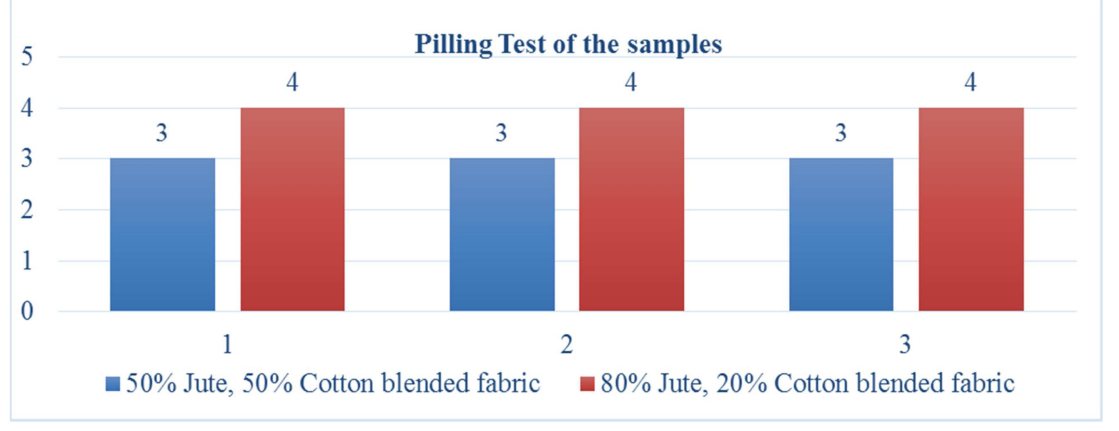

Figure 4. Results of Pilling resistance of the samples.

\subsection{Determination of Drape Co-efficient of Samples}

The drape is measured as the drape co-efficient which is the ratio of Total mass of paper ring to Mass of shaded area expressed in percentage i.e.

Drape co efficient $=$ (Total mass of paper ring $/$ Mass of shaded area) x 100\%. From tables 4 and 5, it is clear that drape co efficient of $80 \%$ Jute \& $20 \%$ Cotton blended woven fabric is higher than drape co efficient of $50 \%$ Jute \& $50 \%$ Cotton blended woven fabric. It is known that the higher drape co efficient, lower the drape ability of the fabric. So, $50 \%$ Jute \& $50 \%$ Cotton blended woven fabric possess lower drape resistance than $80 \%$ jute and $20 \%$ cotton blended samples. This is due to jute fibre process higher strength than cotton fibre.

Table 4. Results of drape co efficient of 50\% Jute \& 50\% Cotton blended woven fabric.

\begin{tabular}{llll}
\hline Total mass of paper ring & Mass of shaded area & Drape co efficient & Average \\
\hline 4.38 & 2.85 & $65.07 \%$ & $65.15 \%$ \\
4.39 & 2.86 & $65.15 \%$ & $65.36 \%$ \\
4.39 & 2.87 & & \\
\hline
\end{tabular}

Table 5. Results of drape co efficient of $80 \%$ Jute \& $20 \%$ Cotton blended woven fabric.

\begin{tabular}{llll}
\hline Total mass of paper ring & Mass of shaded area & Drape co efficient & Average \\
\hline 4.39 & 3.28 & $74.72 \%$ & $74.70 \%$ \\
4.38 & 3.27 & $74.66 \%$ & $74.72 \%$ \\
4.39 & 3.28 & & \\
\hline
\end{tabular}

\subsection{Results of Colour Measurement}

Color strength of sample 50\% Jute \& 50\% Cotton blended woven fabric and $80 \%$ Jute \& $20 \%$ Cotton blendedwoven fabricare measured through $\mathrm{K} / \mathrm{S}$ value. When reflectance is more, absorbance is less which indicates the $\mathrm{K} / \mathrm{S}$ value is less.
On the other hand when reflectance is less; absorbance is more, indicates the more $\mathrm{K} / \mathrm{S}$ value. $\mathrm{K} / \mathrm{S}$ value was measured in $360 \mathrm{~nm}-700 \mathrm{~nm}$ wave lengths. For $50 \%$ Jute \& 50\% Cotton blended woven fabric value of $\mathrm{K} / \mathrm{S}$ is 4.669 at $360 \mathrm{~nm}$ and 4.425 at $700 \mathrm{~nm}$. For $80 \%$ Jute \& $20 \%$ Cotton blended woven fabric K/S was 4.4770 at $360 \mathrm{~nm}$ and 4.435 at $700 \mathrm{~nm}$ wave length. 


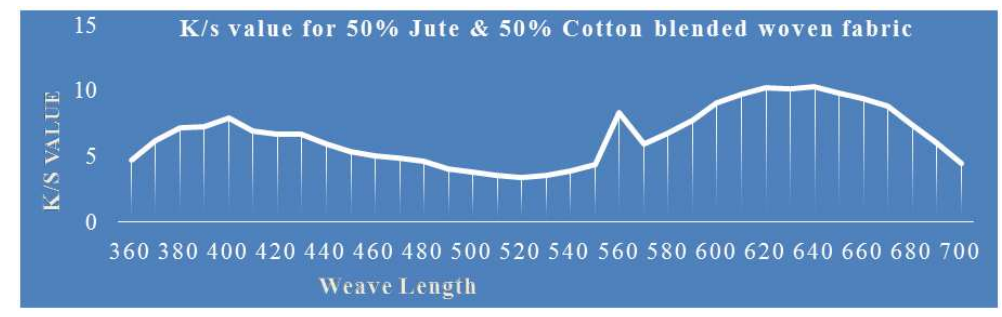

Figure 5. $\mathrm{K} / \mathrm{s}$ value for $50 \%$ Jute \& $50 \%$ Cotton blended woven fabric.

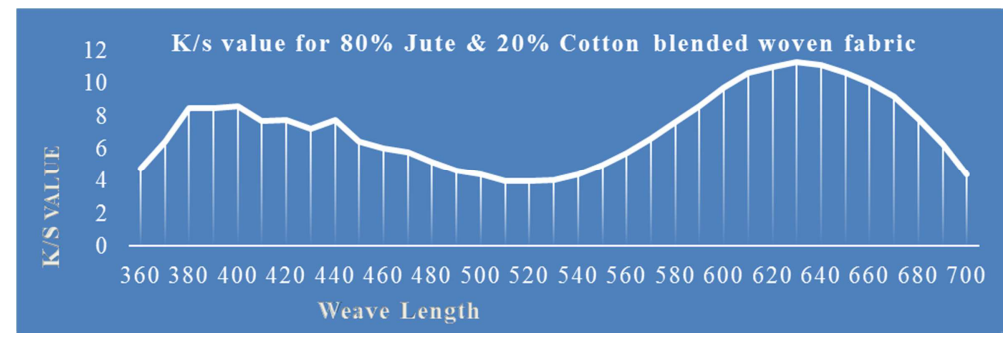

Figure 6. K/s value for $80 \%$ Jute \& $20 \%$ Cotton blended woven fabric.

\subsection{Determination of Water Takes Up Percentage}

From tables 6 and 7 , it is seen that water take up $\%$ for $50 \%$ Jute \& $50 \%$ Cotton blended woven fabric is greater than $80 \%$ Jute \& $20 \%$ Cotton blended woven fabric because of higher jute percentage.

Table 6. Results of water take up $\%$ of $50 \%$ Jute \& $50 \%$ Cotton blended woven fabric.

\begin{tabular}{llll}
\hline & Wet weight & Water take up \% & Average \\
\hline $0.330 \mathrm{gm}$ & $0.901 \mathrm{gm}$ & $63.37 \%$ & \\
$0.331 \mathrm{gm}$ & $0.902 \mathrm{gm}$ & $63.33 \%$ & $63.39 \%$ \\
$0.329 \mathrm{gm}$ & $0.901 \mathrm{gm}$ & $63.48 \%$ & \\
\hline
\end{tabular}

Table 7. Results of water take up $\%$ of $80 \%$ Jute \& $20 \%$ Cotton blended woven fabric.

\begin{tabular}{llll}
\hline Dry weight & Wet weight & Water take up \% & Average \\
\hline $0.529 \mathrm{gm}$ & $1.179 \mathrm{gm}$ & $55.13 \%$ & \\
$0.531 \mathrm{gm}$ & $1.180 \mathrm{gm}$ & $55.00 \%$ & $55.05 \%$ \\
$0.530 \mathrm{gm}$ & $1.179 \mathrm{gm}$ & $55.04 \%$ & \\
\hline
\end{tabular}

\section{Conclusion}

Through this research it was analysis the effects of jute fibres percentage variation on Cotton Jute Blended woven fabric after dyeing process was characterized on weave design, yarn count, fabric density, cover factor, weight per unit area $\left(\mathrm{g} / \mathrm{m}^{2}\right)$, fabric strength, dyeing performances such as wash and rubbing fastness, drape ability, pilling resistance, reflectance and color strength. The study expresses that, the characteristics of blended yarn fabric can be used as fully cotton fabric which may reduce the dependability on importable cotton fiber and may reduce the dependability on $100 \%$ cotton yarn. By enhancing the value addition due to locally produced cheaper jute as a raw material, an opportunity to integrate the blended yarn into the production of jute cotton denim and other heavy fabrics too. If the principle of this research can be applied in textile production purposes, it will be more beneficial to the textile sector.

\section{References}

[1] Talukdar, M. K., Sriramulu, P. K., \& Ajgaonkar, D. B. (1998). Weaving: Machines, Mechanisms, Management. Mahajan Publishers.

[2] Shahid, M. A., Ahmed, F., \& Mahabubuzzaman, A. K. M. (2012). Study on the Physical Properties of Jute-Cotton Blended Rotor Yarn. SUST Journal of Science and Technology, 20 (6), 84-88.

[3] Salam, M. A., Farouqui, F. I., \&Mondal, M. I. (2007). A Study on Sulphonated Jute-cotton Blended Yarn and Fabrics Jute-cotton Blended Yarn and Fabrics and their Characteristics. Bangladesh Journal of Scientific and Industrial Research, 42 (3), 281-286.

[4] Charankar, S. P., Verma, V., \& Gupta, M. (2007). Growing importance of cotton blends in apparel market. Journal of the Textile Association, 67 (5), 201-210.

[5] ISO 13937-2: 2000, Textiles-Tear properties of fabrics-Part 2: Determination of tear force of trouser-shaped test specimens (Single tear method).

[6] Yuen, C. W. M., S. K. A. Ku, P. S. R. Choi, and C. W. Kan. "Study of the factors influencing colour yield of an ink - jet printed cotton fabric." Coloration technology 120 , no. 6 (2004): 320-325.

[7] Prabu, H. Gurumallesh, and M. Sundrarajan*. "Effect of the bio - salt trisodium citrate in the dyeing of cotton." Coloration technology 118, no. 3 (2002): 131-134.

[8] Phillips, Duncan, Geoff Bevan, John Lloyd, Robin Hall, and Jurgen Hoffmeister. "The colour fastness of dyed fabrics to activated oxygen bleach-containing detergents: an international interlaboratory trial." Coloration Technology 115, no. 3 (1999): 100-101.

[9] ASTM D4966-98, Standard Test Method for Abrasion Resistance of Textile Fabrics (Martindale Abrasion Tester Method). 
[10] Cusick, G. E. "21-the measurement of fabric drape." Journal of the Textile Institute 59, no. 6 (1968): 253-260.

[11] Sarkar, Ajoy K., and Corinne M. Seal. "Color strength and colorfastness of flax fabrics dyed with natural colorants." Clothing and Textiles Research Journal 21, no. 4 (2003): 162-166.
[12] Solaiman, J. S. (2015). Comparative analysis of manual fading and laser fading process on denim fabric. Science Discovery, 3 (6), 44-49.

[13] Karmaker, A. C., A. Hoffmann, and G. Hinrichsen. "Influence of water uptake on the mechanical properties of jute fiber-reinforced polypropylene." Journal of Applied Polymer Science 54, no. 12 (1994): 1803-1807. 